\title{
Tuberculosis Treatment Outcomes Among HIV/TB-Coinfected Children in the International Epidemiology Databases to Evaluate AIDS (leDEA) Network
}

\author{
James G. Carlucci, MD, ${ }^{*}$ Meridith Blevins Peratikos, MS, * $\neq$ Aaron M. Kipp, PhD, * $\xi \|$ \\ Mary L. Lindegren, MD, MPH, * Quy T. Du, MD,\# Lorna Renner, MD, ** Gary Reubenson, MBBCh, †† \\ John Ssali, MD, $\neq$ Marcel Yotebieng, MD, PhD, MPH, $\S$ Anna M. Mandalakas, MD, ||

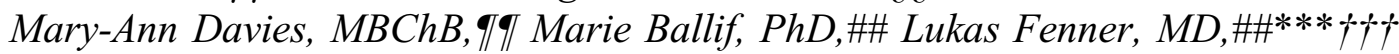 \\ April C. Pettit, MD, MPH, $\$+t+$ \\ and for the International Epidemiology Databases to Evaluate AIDS (IeDEA) Network
}

\begin{abstract}
Introduction: Management of tuberculosis (TB) is challenging in HIV/TB-coinfected children. The World Health Organization recommends nucleic acid amplification tests for TB diagnosis, a 4-drug regimen including ethambutol during intensive phase (IP) of treatment, and initiation of antiretroviral therapy (ART) within 8 weeks of TB diagnosis. We investigated TB treatment outcomes by diagnostic modality, IP regimen, and ART status.
\end{abstract}

Methods: We conducted a retrospective cohort study among HIV/ TB-coinfected children enrolled at the International Epidemiology Databases to Evaluate AIDS treatment sites from 2012 to 2014. We modeled TB outcome using multivariable logistic regression including diagnostic modality, IP regimen, and ART status.

Results: Among the $386 \mathrm{HIV}$-infected children diagnosed with TB, 20\% had microbiologic confirmation of $\mathrm{TB}$, and $20 \%$ had unfavorable TB outcomes. During IP, $78 \%$ were treated with a 4-drug regimen. Thirty-one percent were receiving ART at the time of TB diagnosis, and $32 \%$ were started on ART within
8 weeks of TB diagnosis. Incidence of ART initiation within 8 weeks of TB diagnosis was higher for those with favorable TB outcomes $(64 \%)$ compared with those with unfavorable outcomes $(40 \%)(P=0.04)$. Neither diagnostic modality (odds ratio 1.77; $95 \%$ confidence interval: 0.86 to 3.65 ) nor IP regimen (odds ratio 0.88 ; $95 \%$ confidence interval: 0.43 to 1.80 ) was associated with TB outcome.

Discussion: In this multinational study of HIV/TB-coinfected children, many were not managed as per World Health Organization guidelines. Children with favorable TB outcomes initiated ART sooner than children with unfavorable outcomes. These findings highlight the importance of early ART for children with HIV/TB coinfection, and reinforce the need for implementation research to improve pediatric TB management.

Key Words: tuberculosis, HIV, pediatrics, developing countries, treatment outcome

(J Acquir Immune Defic Syndr 2017;75:156-163)

Received for publication September 8, 2016; accepted February 6, 2017.

From the *Vanderbilt Institute for Global Health, Nashville, TN; $\uparrow$ Division of Pediatric Infectious Diseases, Department of Pediatrics, Vanderbilt University Medical Center, Nashville, TN; †Department of Biostatistics, Vanderbilt University School of Medicine, Nashville, TN; §Vanderbilt Tuberculosis Center, Nashville, TN; $\|$ Division of Epidemiology, Department of Medicine, Vanderbilt University Medical Center, Nashville, TN; $q$ Department of Pediatrics, Vanderbilt University Medical Center, Nashville, TN; \#Children's Hospital 1, Ho Chi Minh City, Vietnam; **University of Ghana School of Medicine and Dentistry, Accra, Ghana; $\dagger \dagger$ Department of Pediatrics and Child Health, Rahima Moosa Mother and Child Hospital, Faculty of Health Sciences, University of the Witwatersrand, Johannesburg, South Africa; țMasaka Regional Referral Hospital, Masaka, Uganda; $\$ \S T h e$ Ohio State University, College of Public Health, Columbus, OH; ||||Department of Pediatrics, Baylor

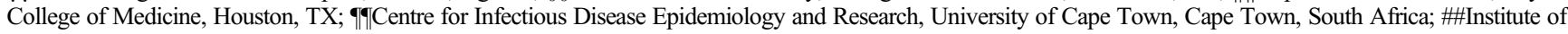
Social and Preventive Medicine, University of Bern, Bern, Switzerland; ***Swiss Tropical and Public Health Institute, Basel, Switzerland; †† $\dagger$ University of Basel, Basel, Switzerland; and †t+Division of Infectious Diseases, Department of Medicine, Vanderbilt University Medical Center, Nashville, TN.

Supported by the National Institutes of Health (NIH) under award numbers: K08 AI104352 (A.C.P.), T32 HD060554-06A1 (J.G.C.), U01 AI096299 (IeDEA Central Africa), U01 AI069919 (IeDEA Western Africa), U01 AI069924 (IeDEA Southern Africa), U01 AI069911 (IeDEA Eastern Africa), U01 AI069907 (IeDEA Asia-Pacific), U01 AI096186 (IeDEA Network Coordinating Center), P30 AI1 10527 (Tennessee Center for AIDS Research), and UL1 TR000445 (Vanderbilt Institute for Clinical and Translational Research). It has also been supported by the President's Emergency Plan for AIDS Relief (PEPFAR) through the United States Agency for International Development (USAID) under the terms of Cooperative Agreement No. AID-623-A-120001.

A preliminary version of this report was presented as a poster at the Conference on Retroviruses and Opportunistic Infections; February 23, 2016; Boston, MA; Abstract \#16-1151. The manuscript is not being considered for publication elsewhere.

The authors have no conflicts of interest to disclose.

The contents of this publication are the sole responsibility of the authors and do not necessarily reflect the views of the NIH, PEPFAR, or USAID.

Correspondence to: James G. Carlucci, MD, Vanderbilt Institute for Global Health, D-7235 Medical Center North, 1161 21st Avenue South, Nashville, TN 37232-2581 (e-mail: james.g.carlucci@vanderbilt.edu).

Copyright $(2017$ Wolters Kluwer Health, Inc. All rights reserved. 


\section{INTRODUCTION}

Tuberculosis (TB) remains a major cause of childhood morbidity and mortality in low- and middle-income countries (LMICs). HIV has amplified the TB epidemic, as evidenced by the disproportionate impact of TB in areas with high HIV prevalence, especially Sub-Saharan Africa and Southeast Asia. ${ }^{1-3}$ Among children in 2014, there were an estimated 1 million incident TB cases, 140,000 deaths attributable to TB, and approximately $40 \%$ of TB deaths were among those with HIV/TB coinfection. ${ }^{1}$

The World Health Organization (WHO) defines TB treatment success as documented cure or completion of antiTB therapy (ATT); unsuccessful TB treatment outcomes include death, treatment failure, default from care/loss to follow-up (LTFU), or unknown outcome. ${ }^{4}$ Compared with HIV-negative individuals, HIV/TB-coinfected individuals are less likely to have successful TB treatment outcomes. ${ }^{1}$

Despite limited data on pediatric TB outcomes, ${ }^{3,5-11}$ WHO has developed guidelines for the management of HIV/ TB-coinfected children in LMICs. ${ }^{6}$ These recommendations include nucleic acid amplification testing (NAAT) as the initial TB diagnostic test for children with HIV-associated $\mathrm{TB}^{6,12}$; using a 4-drug regimen including ethambutol during the intensive phase (IP; first 2 months) of ATT in TBendemic areas with background isoniazid (INH) resistance ${ }^{6,13,14}$; antiretroviral therapy (ART) initiation within 8 weeks of starting ATT, for ART-naive patients ${ }^{6}$; and, for those already receiving ART at the time of TB diagnosis, to optimize ART regimens to avoid drug-drug interactions. ${ }^{6,15}$

However, it is unclear to what extent these recommendations have been implemented in pediatric ART programs in LMICs. Limited availability of diagnostics, challenges with obtaining sputum, and paucibacillary disease which may be more pronounced in HIV/TB-coinfected children may result in many children being diagnosed with TB on clinical criteria alone [without microbiologic confirmation with NAAT, mycobacterial culture, and/or acid-fast bacilli (AFB) smear]. ${ }^{12,16-19}$ Despite studies demonstrating low risk for ethambutol-associated ocular toxicity, ${ }^{13,14}$ some practitioners still opt to prescribe a 3-drug IP regimen lacking ethambutol for preverbal children. Similarly, some providers delay ART initiation beyond the recommended 8 weeks after ATT initiation to avoid immune reconstitution inflammatory syndrome, which is uncommon among children. ${ }^{20,21}$ And, in the setting of medication stock-outs, ${ }^{22-24}$ limited availability of fixed-dose combination drug options, ${ }^{25}$ and adherence challenges with pediatric formulations of ART and ATT medications, ${ }^{26,27}$ optimizing regimens for potential ARTATT interactions can be challenging. ${ }^{15,28}$

We set out to investigate these issues using data collected from sites participating in the International Epidemiology Databases to Evaluate AIDS (IeDEA). ${ }^{29}$ The objectives of this research are to (1) provide recent patientlevel data on the care and treatment of HIV/TB-coinfected children; (2) assess whether TB treatment outcomes differ based on TB diagnostic modality, IP regimen, or ART status; and (3) describe ART regimens and modifications made to ART regimens in the setting of ATT.

\section{METHODS}

\section{Study Design and Population}

We conducted a retrospective cohort study among HIV/ TB-coinfected children aged 0 through 15 years. Children were enrolled from 14 participating ART programs in 11 LMICs, representing 5 IeDEA regions (Fig. 1). ${ }^{29}$ Previously treated TB cases were included $(n=8)$, so a patient could contribute more than one TB episode.

\section{Data Collection}

Local TB registries were used to identify TB cases diagnosed at participating IeDEA sites from January 2012 through December 2014. Patient demographics, laboratory data, TB treatment regimens, and TB outcomes were collected retrospectively using a standardized electronic case report form (CRF) developed in Research Electronic Data Capture (REDCap). ${ }^{30}$ The CRF was piloted before implementation, and was made available in English and French. The French version was translated by an IeDEA coinvestigator who is a native French speaker and engaged in HIV/TB research. Local IeDEA site investigators completed CRFs for TB cases after electronic and/or hard copy medical record review. Data entry took place from January 2012 through January 2016. Information on ART initiation dates and ART regimens were obtained from IeDEA regional HIV care and treatment data repositories for all patients with a completed CRF. Throughout the period of data collection, routine audits were made to ensure data quality. After database closure, the data were further verified, and any suspected data quality issues were referred back to local sites for investigation, clarification, and revision when necessary.

\section{Definitions}

Any diagnosis of new or previously treated active TB while enrolled at one of the participating ART sites was considered a unique TB episode. Using WHO definitions, ${ }^{4}$ we grouped TB treatment outcomes into favorable and unfavorable categories. Documented cure or completion of ATT were considered favorable TB treatment outcomes. Death, treatment failure, default from care/LTFU, or unknown outcome were considered unfavorable TB treatment outcomes-the primary outcome of interest. Children still on ATT at database closure or who transferred care to another facility were excluded from the analysis of TB treatment outcomes.

Our primary exposure variables of interest were diagnostic modality, IP regimen, and ART status. A microbiologic diagnosis was considered to be any diagnosis of TB that was confirmed by at least 1 laboratory test (AFB smear, mycobacterial culture, or NAAT). A clinical diagnosis was any TB diagnosis that was made without a positive laboratory test result; children with clinical diagnoses could have had negative diagnostic test result(s) or no diagnostic testing performed. All TB diagnoses included a decision to start ATT.

IP regimen was dichotomized by those who received the recommended 4-drug IP regimen [INH $(\mathrm{H})$, rifampin $(\mathrm{R})$, 


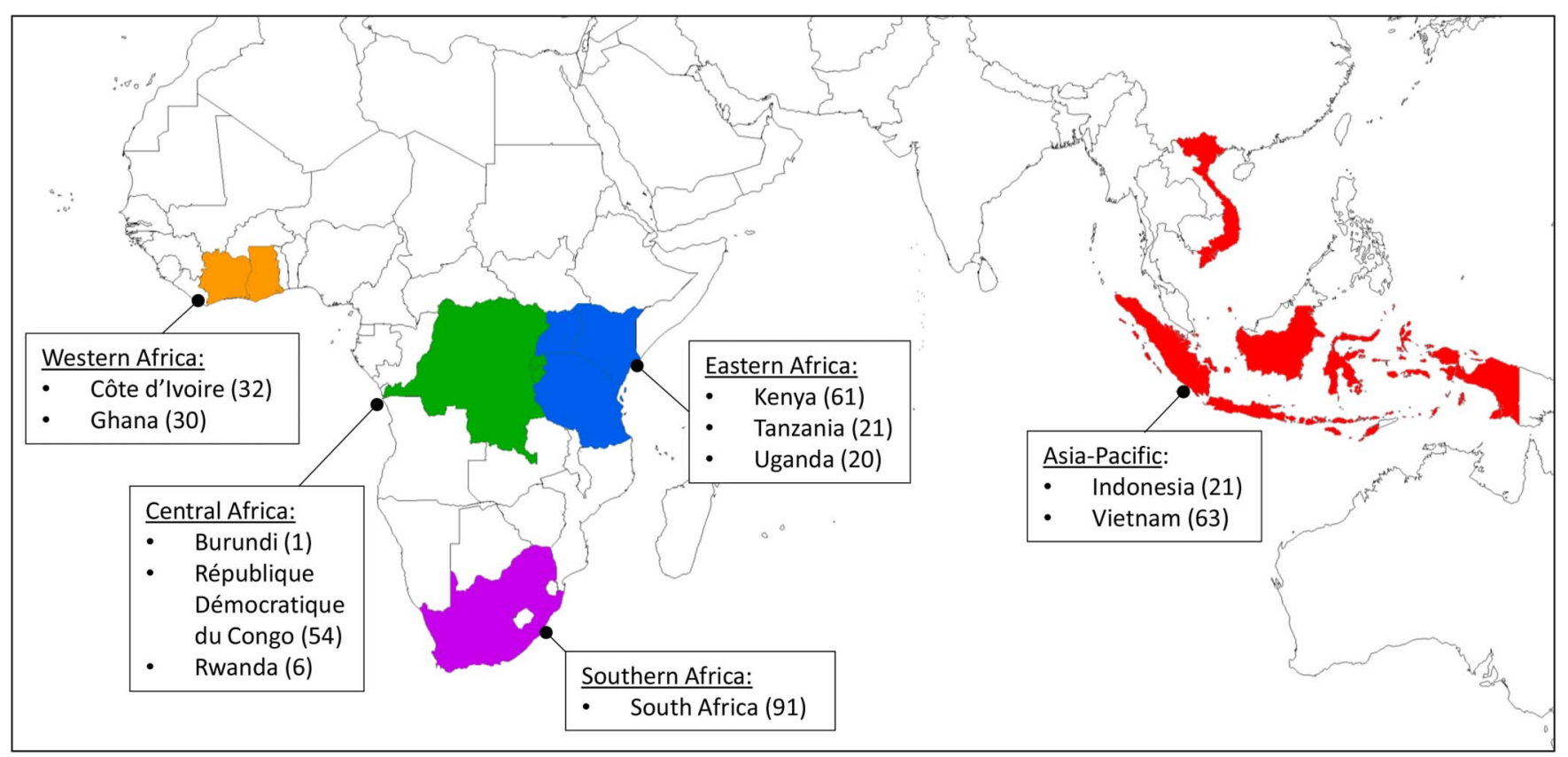

FIGURE 1. HIV/TB-coinfected children included in the study, by leDEA Network region and country. Numbers in parentheses indicate the number of children contributed by each country.

pyrazinamide $(Z)$, ethambutol (E); HRZE] and those who received a 3-drug IP regimen lacking ethambutol (HRZ).

ART status at the time of TB diagnosis was categorized as ART naive or previously on ART. Among those who were ART naive at the time of TB diagnosis, we also calculated time to ART initiation from TB diagnosis by subtracting the date of ATT initiation from ART initiation date. ART regimens were classified into the following categories based on the WHO's recommendations ${ }^{31}$ : 3 nucleoside reversetranscriptase inhibitors (NRTIs); 2 NRTIs plus a non-NRTI (NNRTI); 2 NRTIs plus a boosted protease inhibitor (PI), and nonstandard ("other") regimens. ART regimen modifications made within 8 weeks of TB diagnosis date were included as ART modifications made in the setting of ATT initiation.

Key covariates at TB diagnosis included age, sex, weight, CD4 count (and CD4 percentage), and IeDEA region (Asia-Pacific, Central Africa, Eastern Africa, Southern Africa, and Western Africa; Fig. 1). The date of TB diagnosis was defined as the date of the first positive microbiologic test or clinical diagnosis of TB. CD4 count at TB diagnosis was defined as the first available value 180 days before or up to 30 days after the date of TB diagnosis. HIV viral loads were not routinely collected or reported at some treatment sites participating in this study. Weight-for-age Z-scores (WAZ) at $\mathrm{TB}$ diagnosis were calculated as a marker of nutritional status using United States Centers for Disease Control (CDC) standards. $^{32}$ WHO standards are more commonly used to calculate WAZ in LMICs because the reference population from which they are derived is more diverse than the CDC reference population; however, the WHO standard only extends through 10 years of age, whereas the CDC standard extends through 18 years. Therefore, to maintain a consistent metric for our population of children $0-15$ years of age, we

158 | www.jaids.com elected to use the CDC standard. Regardless of the reference population, WAZ is a relative measure and lower WAZ scores indicate a greater degree of undernutrition.

\section{Statistical Analyses}

Descriptive statistics were used to summarize patient characteristics, TB diagnostics, TB treatment, TB outcomes, ART status, ART regimens, and modifications made to ART regimens in the setting of TB diagnosis and treatment. For continuous variables, median and interquartile ranges (IQRs) are reported. Frequency and percentages are reported for categorical variables.

Multivariable logistic regression was used to estimate the odds ratio (OR) and 95\% confidence interval (CI) of unfavorable TB treatment outcome associated with mode of diagnosis, IP regimen, and ART status while adjusting for age, sex, WAZ, CD4 count/percentage, and IeDEA region. Multiple imputation was used to account for missing data. Continuous variables were modeled as linear terms as there was no evidence for nonlinearity. Acknowledging that there may be important differences between diagnostic and treatment strategies for younger children, an a priori decision was made to perform secondary regression analyses for the subgroups of children younger than 5 years and children aged 5-15 years. The same covariates were included in main and secondary models, except that CD4 percentage was used instead of CD4 count for those younger than 5 years. Sensitivity analyses excluding the small number of previously treated TB cases were also performed.

Among those who were ART naive at TB diagnosis, we compared ART initiation across TB outcomes. Anticipating that LTFU during the 8 weeks after TB diagnosis might make 
it difficult to investigate the effect of ART initiation during the first 8 weeks, we plotted the cumulative incidence of ART initiation among groups with unfavorable and favorable TB treatment outcome restricted to those that were alive and in care at 8 weeks after TB diagnosis, and tested the association using a log-rank test.

$\mathrm{R}$ version 3.2.5 (www.r-project.org) was used for all analysis and code is available online (http://biostat.mc. vanderbilt.edu/ArchivedAnalyses).

\section{Ethical Considerations}

Local institutional review board or ethics committee approval was obtained from all local study sites as well as Vanderbilt University. Procedures followed were in accordance with the ethical standards of the responsible committee on human experimentation (institutional and national) and with the Helsinki Declaration of 1975, as revised in 2013.

\section{RESULTS}

\section{Patient Characteristics}

There were 400 children younger than the age of 16 diagnosed with TB and with a TB CRF entered into REDCap during the study period. Fourteen $(3.5 \%)$ were excluded; one was still on ATT and 13 had transferred to another facility. Among 386 children included, the median age was 5.7 years (IQR 2.0-9.5) and 47\% were females. Median WAZ was -2.69 (IQR -3.82 to $-1.48 ; 17 \%$ missing data). Median CD4 count among those $\geq 5$ years was 144 cells $/ \mathrm{mm}^{3}$ (IQR
$28-403$ cells $/ \mathrm{mm}^{3} ; 14 \%$ missing data). Median CD4 percentage among those $<5$ years was $16 \%$ (IQR $8 \%-23 \%$; 34\% missing data) (Table 1). The children were recruited from 14 ART programs in 11 LMICs (Burundi, Côte d'Ivoire, Ghana, Indonesia, Kenya, République Démocratique du Congo, Rwanda, South Africa, Tanzania, Uganda, and Vietnam), each contributing between 1 and 91 children (Fig. 1).

\section{TB Diagnosis}

Among the $386 \mathrm{HIV} / \mathrm{TB}-$ coinfected children, $79(20 \%)$ had microbiologic confirmation of TB, whereas $307(80 \%)$ were diagnosed clinically. Of the 79 with at least 1 positive diagnostic test, $36(46 \%)$ had a positive AFB smear, 33 (42\%) had a positive mycobacterial culture, and 48 (61\%) had a positive NAAT (Table 2). In terms of diagnostic yield, 59\% (229/386) of cases were tested with AFB smear, but only $16 \%$ $(36 / 229)$ of those tests were positive; $32 \%(123 / 386)$ of cases were tested with mycobacterial culture, and $27 \%(33 / 123)$ of those tests were positive; whereas only $22 \%(84 / 386)$ of cases were tested with NAAT, but $57 \%(48 / 84)$ of those tests were positive. Of the 307 cases diagnosed clinically, $155(50 \%)$ had at least 1 test but no positive result, and $152(50 \%)$ did not have a laboratory diagnostic test performed. Overall, 287 $(74 \%)$ of cases were classified as pulmonary TB, $73(19 \%)$ were classified as extrapulmonary TB (EPTB), and $25(6 \%)$ were classified as having both pulmonary TB and EPTB. Among those with EPTB, the most common sites were abdominal $(9 \%)$ and lymphatic (7\%) (Table 2).

Only 3 cases $(<1 \%)$ were found to have INH or rifampin resistance, one of which was in the Asia-Pacific

TABLE 1. Characteristics of HIV/TB-Coinfected Children at the Time of TB Diagnosis, Stratified by TB Treatment Outcome, 2012-2014

\begin{tabular}{|c|c|c|c|c|c|c|c|c|c|}
\hline & \multicolumn{3}{|c|}{ Favorable Outcome } & \multicolumn{5}{|c|}{ Unfavorable Outcome } & \multirow{2}{*}{$\begin{array}{l}\text { Combined } \\
(N=\mathbf{3 8 6})\end{array}$} \\
\hline & $\begin{array}{c}\text { Completed } \\
\text { ATT } \\
(n=240)\end{array}$ & Cure $(n=68)$ & $\begin{array}{c}\text { All } \\
\text { Favorable } \\
(n=308)\end{array}$ & $\begin{array}{c}\text { Death } \\
(n=30)\end{array}$ & $\begin{array}{c}\text { Treatment } \\
\text { Failure } \\
(n=1)\end{array}$ & $\begin{array}{c}\text { LTFU } \\
(n=33)\end{array}$ & $\begin{array}{c}\text { Unknown } \\
(n=14)\end{array}$ & $\begin{array}{c}\text { All } \\
\text { Unfavorable } \\
(n=78)\end{array}$ & \\
\hline Age, median (IQR) & $6(2.1-9.8)$ & $5.5(2.7-9.4)$ & $5.9(2.4-9.6)$ & $5.4(2-9.8)$ & $10.2(10.2-10.2)$ & $3.6(1-6.7)$ & $8.1(6.6-9.6)$ & $5.5(1.1-8.8)$ & $5.7(2.0-9.5)$ \\
\hline \multicolumn{10}{|l|}{ Age group, n (\%), yrs } \\
\hline $0-4.9$ & $100(42)$ & $29(43)$ & $129(42)$ & $13(43)$ & $0(0)$ & $19(58)$ & $3(21)$ & $35(45)$ & $164(43)$ \\
\hline $5-9.9$ & $80(33)$ & $25(37)$ & $105(34)$ & $9(30)$ & $0(0)$ & $10(30)$ & $8(57)$ & $27(35)$ & $132(34)$ \\
\hline $10-15$ & $60(25)$ & $14(21)$ & $74(24)$ & $8(27)$ & $1(100)$ & $4(12)$ & $3(21)$ & $16(21)$ & $90(23)$ \\
\hline Female, n (\%) & $121(50)$ & $28(41)$ & $149(48)$ & $13(43)$ & $1(100)$ & $11(33)$ & $7(50)$ & $32(41)$ & $181(47)$ \\
\hline WAZ, median (IQR) & $\begin{array}{c}-2.33 \\
(-3.57 \text { to }-1.11)\end{array}$ & $\begin{array}{c}-2.91 \\
(-3.76 \text { to }-1.98)\end{array}$ & $\begin{array}{c}-2.45 \\
(-3.66 \text { to }-1.36)\end{array}$ & $\begin{array}{c}-4.36 \\
(-5.13 \text { to }-2.45)\end{array}$ & $\begin{array}{c}-4.25 \\
(-4.25 \text { to }-4.25)\end{array}$ & $\begin{array}{c}-2.64 \\
(-3.54 \text { to }-1.81)\end{array}$ & $\begin{array}{c}-3.75 \\
(-4.89 \text { to }-2.94)\end{array}$ & $\begin{array}{c}-3.26 \\
(-4.84 \text { to }-2.18)\end{array}$ & $\begin{array}{c}-2.69 \\
(-3.82 \text { to }-1.48)\end{array}$ \\
\hline Missing, n (\%) & $45(19)$ & $6(9)$ & $51(17)$ & $5(17)$ & $0(0)$ & $7(21)$ & $4(29)$ & $16(21)$ & $67(17)$ \\
\hline $\begin{array}{l}\text { CD4 count*, median } \\
\text { (IQR) }\end{array}$ & $262(70-665)$ & $117(16-531)$ & $229(54-638)$ & $145(63-324)$ & $92(92-92)$ & $670(367-890)$ & $85(20-268)$ & $267(81-670)$ & $247(60-668)$ \\
\hline Missing, n (\%) & $55(23)$ & $2(3)$ & $57(19)$ & $8(27)$ & $0(0)$ & $10(30)$ & $3(21)$ & $21(27)$ & $78(20)$ \\
\hline $\begin{array}{l}\mathrm{CD} 4 \% \dagger, \text { median } \\
\text { (IQR) }\end{array}$ & $12(4-22)$ & $5(1-18)$ & $10(3-22)$ & $7(2-13)$ & $11(11-11)$ & $17(10-22)$ & $7(4-10)$ & $10(5-17)$ & $10(3-20)$ \\
\hline Missing, n (\%) & $75(31)$ & $7(10)$ & $82(27)$ & $8(27)$ & $0(0)$ & $11(33)$ & $3(21)$ & $22(28)$ & $104(27)$ \\
\hline \multicolumn{10}{|l|}{ IeDEA region, $\mathrm{n}(\%)$} \\
\hline Asia-Pacific & $17(7)$ & $53(78)$ & $70(23)$ & $13(43)$ & $0(0)$ & $1(3)$ & $0(0)$ & $14(18)$ & $84(21)$ \\
\hline Central Africa & $52(22)$ & $1(1)$ & $53(17)$ & $3(10)$ & $0(0)$ & $4(12)$ & $0(0)$ & $7(9)$ & $60(15)$ \\
\hline Eastern Africa & $60(25)$ & $9(13)$ & $69(22)$ & $10(33)$ & $0(0)$ & $3(9)$ & $12(86)$ & $25(32)$ & $94(26)$ \\
\hline Southern Africa & $76(32)$ & $1(1)$ & $77(25)$ & $1(3)$ & $0(0)$ & $8(24)$ & $2(14)$ & $11(14)$ & $88(23)$ \\
\hline Western Africa & $35(15)$ & $4(6)$ & $39(13)$ & $3(10)$ & $1(100)$ & $17(52)$ & $0(0)$ & $21(27)$ & $60(16)$ \\
\hline
\end{tabular}

*Median CD4 count among those $\geq 5$ years was 144 cells $/ \mathrm{mm}^{3}$ (IQR $28-403$ cells $/ \mathrm{mm}^{3} ; 14 \%$ missing data).

$\dagger$ Median CD4 percentage among those $<5$ years was $16 \%$ (IQR $8 \%-23 \%$; 34\% missing data). 
TABLE 2. Summary of TB Diagnostics, Sites of Disease, Treatment, and ART Status for HIV/TB-Coinfected Children, Stratified by TB Treatment Outcome Category

\begin{tabular}{|c|c|c|c|}
\hline & $\begin{array}{c}\text { Favorable } \\
\text { Outcome } \\
(n=308)\end{array}$ & $\begin{array}{c}\text { Unfavorable } \\
\text { Outcome } \\
(\mathbf{n}=78) \\
\end{array}$ & $\begin{array}{r}\text { Combined } \\
(\mathbf{N}=\mathbf{3 8 6}) \\
\end{array}$ \\
\hline \multicolumn{4}{|l|}{ Test Result, n (\%) } \\
\hline Microbiologic diagnosis* & $61(20)$ & $18(23)$ & $79(20)$ \\
\hline AFB smear positive & $25(8)$ & $11(14)$ & $36(9)$ \\
\hline AFB culture positive & $28(9)$ & $5(6)$ & $33(9)$ \\
\hline NAAT positive & $37(12)$ & $11(15)$ & $48(12)$ \\
\hline Clinical diagnosis & $247(80)$ & $60(77)$ & $307(80)$ \\
\hline Negative test result(s) & $127(41)$ & $28(36)$ & $155(40)$ \\
\hline No test performed & $120(39)$ & $32(41)$ & $152(39)$ \\
\hline \multicolumn{4}{|l|}{ Site of disease, $\mathrm{n}(\%)$} \\
\hline Pulmonary & $229(74)$ & $58(74)$ & $287(74)$ \\
\hline Extrapulmonary* & $60(19)$ & $13(17)$ & 73 (19) \\
\hline Abdominal & $30(10)$ & $5(6)$ & $35(9)$ \\
\hline $\mathrm{CNS} /$ meningeal & $3(1)$ & $1(1)$ & $4(1)$ \\
\hline Lymphatic & $16(5)$ & $9(12)$ & $25(7)$ \\
\hline Miliary & $4(1)$ & $2(3)$ & $6(2)$ \\
\hline Osteoarticular & $2(1)$ & $0(0)$ & $2(<1)$ \\
\hline Pericardial & $1(<1)$ & $1(1)$ & $2(<1)$ \\
\hline Pleural & $4(1)$ & $3(4)$ & $7(2)$ \\
\hline Unknown & $11(4)$ & $1(1)$ & $12(3)$ \\
\hline Both & $18(6)$ & $7(9)$ & $25(6)$ \\
\hline \multicolumn{4}{|l|}{ IP regimen, $\mathrm{n}(\%) \dagger$} \\
\hline 3-drug HRZ & $62(20)$ & $22(28)$ & $84(22)$ \\
\hline 4-drug HRZE & $246(80)$ & $56(72)$ & $302(78)$ \\
\hline \multicolumn{4}{|l|}{ ART status, n (\%) } \\
\hline ART before TB diagnosis & $101(33)$ & $18(23)$ & $119(31)$ \\
\hline $\begin{array}{l}\text { ART } \leq 8 \text { wks after TB } \\
\text { diagnosis }\end{array}$ & $109(34)$ & $15(19)$ & $124(32)$ \\
\hline $\begin{array}{l}\text { ART }>8 \text { wks after TB } \\
\text { diagnosis }\end{array}$ & $45(15)$ & $8(10)$ & $53(14)$ \\
\hline No ART & $25(8)$ & $25(32)$ & $50(13)$ \\
\hline Missing & $28(9)$ & $12(15)$ & $40(10)$ \\
\hline
\end{tabular}

* More than one can apply, thus numbers do not add up to $100 \%$.

$\dagger$ A 3-drug regimen includes INH (H), rifampin (R), and pyrazinamide (Z). A 4-drug regimen includes HRZ and ethambutol (E).

region, and the other 2 were in the Southern Africa region. These regions were also the only 2 regions that were routinely using NAAT for TB diagnosis, and were the primary regions where gastric aspirates and induced sputum were used to obtain diagnostic specimens.

\section{TB Treatment Regimens}

As for IP regimens, $84(22 \%)$ cases were treated with a nonstandard 3-drug regimen lacking ethambutol, whereas $302(78 \%)$ were treated with a 4-drug regimen including ethambutol (Table 2). The practice of omitting ethambutol was common in Eastern Africa, Central Africa, and Western Africa regions where such IP regimens were prescribed for $47 \%, 36 \%$, and $27 \%$ of cases, respectively. IP regimens lacking ethambutol were prescribed to children younger than 5 years in $32 \%$ of cases, and to children aged 5-15 years in $15 \%$ of cases.
ART Status

One hundred nineteen (31\%) of the $386 \mathrm{HIV} / \mathrm{TB}-$ coinfected children were receiving ART before their TB diagnosis. Of the $267(69 \%)$ not on ART at the time of starting ATT, $124(46 \%)$ initiated ART within 8 weeks of starting ATT, and $53(20 \%)$ initiated ART more than 8 weeks after starting ATT. Fifty (13\%) of the HIV/TB-coinfected children did not initiate ART by the end of the study period. ART status data were missing for $40(10 \%)$ children (Table 2).

Many ART regimens were modified when starting ATT. Among the 114 patients active on ART 8 weeks before TB diagnosis, $64 \%$ of the ART regimens were composed of 2 NRTIs plus an NNRTI (32\% efavirenz and 32\% nevirapine); $16 \%$ were composed of 2 NRTIs plus a boosted PI; $2 \%$ were composed of 3 NRTIs; and 18\% were nonstandard regimens. The majority of children receiving an efavirenz-based regimen $(97 \%)$, boosted PI-based regimen $(94 \%)$, or 3 NRTIs $(100 \%)$ regimen remained on the same ART regimen after TB diagnosis. Sixty percent of children on a nevirapine-based regimen remained on the same regimen after TB diagnosis; $32 \%$ switched to an efavirenz-based regimen; 3\% switched to a boosted PI-based regimen; and 3\% switched to 3 NRTIs. Two percent experienced an interruption in ART after TB diagnosis. Children initiating ART within 8 weeks of TB diagnosis were started on the following regimens: 2 NRTIs plus an NNRTI (78\%; 66\% efavirenz, 12\% nevirapine); 2 NRTIs plus a boosted PI (8\%); 3 NRTIs (2\%); and other nonstandard regimens (12\%) (supplemental figure accessible at: http://biostat.mc.vanderbilt.edu/MeridithBlevins/pediatrictb-hiv-sunburst.html). Dose adjustments for ART and/or ATT also may have occurred, but dosing data were not captured.

\section{TB Treatment Outcomes}

Among the $386 \mathrm{HIV} / \mathrm{TB}$-coinfected children, 78 (20\%) had unfavorable TB treatment outcomes and 308 (80\%) had favorable outcomes. Among the 78 with unfavorable outcomes, $30(38 \%)$ died, $1(1 \%)$ had treatment failure, $33(42 \%)$ were LTFU, and $14(18 \%)$ had unknown outcomes. The proportion of unfavorable outcomes attributable to LTFU was $1 \%(1 / 84)$ in the Asia-Pacific region, $7 \%(4 / 60)$ in the Central Africa region, 3\% (3/94) in the Eastern Africa region, 9\% $(8 / 88)$ in the Southern Africa region, and 28\% (17/60) in the Western Africa region. Among the 308 with favorable TB treatment outcomes, 68 (22\%) had documented cure, and 240 (78\%) completed therapy (Table 1).

Multivariable logistic regression was performed to identify factors independently associated with an unfavorable TB treatment outcome. In the model including all $386 \mathrm{HIV} /$ TB-coinfected children, neither mode of diagnosis (OR 1.77; 95\% CI: 0.86 to 3.65 ), IP regimen (OR 0.88 ; $95 \%$ CI: 0.43 to 1.80 ), nor ART status (OR $0.71 ; 95 \%$ CI: 0.38 to 1.31 ) were significantly associated with TB treatment outcome. Better nutritional status/higher WAZ was protective against unfavorable TB treatment outcomes (OR 0.35; 95\% CI: 0.67 to 0.94 ). Children in the Asia-Pacific (OR 0.35; 95\% CI: 0.14 to 0.89 ) and Southern Africa $(0.34 ; 95 \%$ CI: 0.14 to 0.84$)$ IeDEA regions had significantly lower odds of having an unfavorable 
outcome compared with the Eastern Africa region. In the subgroup analysis restricted to children younger than 5 years, those with microbiologic confirmation of TB had higher odds of unfavorable TB outcome (OR 3.8; 95\% CI: 1.12 to 12.94 ) (Table 3 ). When previously treated TB cases were excluded $(\mathrm{n}=8)$, results were similar (data not shown).

Among HIV/TB-coinfected children who were ART naive at the time of TB diagnosis and were alive and retained in care 8 weeks later, ART initiation within 8 weeks of TB diagnosis was higher for those with favorable TB outcomes (64\%) compared with those with unfavorable TB outcomes $(40 \%)(P=0.04)$ (Fig. 2).

\section{DISCUSSION}

In this large multinational study of HIV/TB-coinfected children, many were not diagnosed or treated as per WHO guidelines. Eighty percent of diagnoses were clinical (without microbiologic confirmation), and NAAT use was infrequent. Nearly one-quarter of patients did not receive a 4-drug IP regimen including ethambutol. Only half of ART-naive children were started on ART within 8 weeks of TB diagnosis, and $13 \%$ of patients were not started on ART during the study period. Overall, $20 \%$ of HIV/TB-coinfected children in this cohort had unfavorable TB treatment outcomes. Those who initiated ART earlier were more likely to have favorable TB treatment outcomes. Among those younger than 5 years, those with microbiologic confirmation of TB had higher odds of unfavorable TB treatment outcomes.

Although at first the finding that microbiologic confirmation of TB is associated with unfavorable TB outcomes among the youngest patients seems counterintuitive, this might be explained in several ways. First, there is likely over-diagnosis of TB among clinical cases (false positives) and therefore better outcomes in this group. Second, more extensive disease and, therefore, higher bacterial burden could both facilitate microbiologic confirmation of TB and be associated with poorer outcomes. Third, awaiting culture results in the absence of NAAT confirmation may have delayed TB treatment and possibly ART initiation, and therefore resulted in a greater likelihood of unfavorable TB treatment outcomes.

This study highlights an urgent need to improve diagnosis of TB among HIV/TB-coinfected children in LMICs. Only $20 \%$ of children diagnosed with TB had microbiologic confirmation of disease, and only $22 \%$ of cases were tested with NAAT. Similarly, in 2012 we conducted a site-level survey of pediatric ART programs in 6 IeDEA regions and found that NAAT was available at about one-third of sites; and among 146 children who developed TB, AFB smear was used for $52 \%$, mycobacterial culture for $17 \%$, and NAAT for only $8 \% .{ }^{19}$ Among the many impediments to TB diagnosis in children is obtaining diagnostic respiratory specimens. Performing early morning gastric aspirates can improve diagnostic yield, but requires hospitalization, which is often not practical in LMICs. Capacity to perform sputum induction in the outpatient setting is growing, and can improve diagnostic yield in children unable to spontaneously expectorate sputum. Even when a good specimen is obtained, access to diagnostics may be limited in LMICs. Furthermore, the yield of diagnostics is low because of paucibacillary disease in children, particularly those with compromised immune systems. ${ }^{12,18}$ Strategies to improve pediatric TB diagnosis should be multifaceted, and might include improving the accuracy of clinical diagnosis, enhancing the implementation and utilization of current and emerging NAAT platforms, ${ }^{1}$ and developing non-sputumbased diagnostics. ${ }^{33-35}$

TABLE 3. Logistic Regression Models to Identify Factors Associated With an Unfavorable TB Treatment Outcome

\begin{tabular}{|c|c|c|c|}
\hline & All Pediatric Patients & Children Younger Than 5 & Children Aged 5-15 \\
\hline & OR $(95 \% \mathrm{CI})^{*}$ & OR $(95 \%$ CI $) \dagger$ & OR $(95 \%$ CI $) \ddagger$ \\
\hline Microbiologic diagnosis (vs. clinical diagnosis) & $1.77(0.86$ to 3.65$)$ & $3.80(1.12$ to 12.90$)$ & $1.45(0.53$ to 4.00$)$ \\
\hline 3-drug HRZ regimen (vs. 4-drug HRZE) $§$ & $0.88(0.43$ to 1.80$)$ & $0.84(0.23$ to 2.99$)$ & $0.91(0.33$ to 2.50$)$ \\
\hline On ART at TB diagnosis (vs. not on ART) & $0.71(0.38$ to 1.31$)$ & $1.04(0.40$ to 2.67$)$ & $0.48(0.21$ to 1.14$)$ \\
\hline Age (per 1 yr) & $0.96(0.89$ to 1.03$)$ & $0.83(0.62$ to 1.13$)$ & $1.01(0.88$ to 1.15$)$ \\
\hline WAZ (per 1 SD) & $0.80(0.67$ to 0.94$)$ & $0.80(0.63$ to 1.02$)$ & $0.78(0.61$ to 1.01$)$ \\
\hline CD4 count (per 10 cells) & $1.00(1.00$ to 1.01$)$ & Omitted $\|$ & $1.00(0.99$ to 1.02$)$ \\
\hline CD4 percentage (per 1 unit) & Omitted $\|$ & $1.00(0.96$ to 1.05$)$ & Omitted $\|$ \\
\hline Female (vs. male) & $0.77(0.45$ to 1.32$)$ & $0.93(0.41$ to 2.11$)$ & $0.64(0.30$ to 1.40$)$ \\
\hline \multicolumn{4}{|l|}{ Region } \\
\hline Eastern Africa (reference) & 1 & 1 & 1 \\
\hline Asia-Pacific & $0.35(0.14$ to 0.89$)$ & $0.28(0.05$ to 1.43$)$ & $0.41(0.12$ to 1.40$)$ \\
\hline Central Africa & $0.47(0.18$ to 1.23$)$ & $0.88(0.22$ to 3.52$)$ & $0.23(0.04$ to 1.21$)$ \\
\hline Southern Africa & $0.34(0.14$ to 0.84$)$ & $0.91(0.23$ to 3.59$)$ & $0.08(0.02$ to 0.45$)$ \\
\hline Western Africa & $1.22(0.57$ to 2.62$)$ & $2.81(0.69$ to 11.53$)$ & $0.78(0.31$ to 1.99$)$ \\
\hline \multicolumn{4}{|c|}{$\begin{array}{l}\text { *There are } 386 \text { patients included in this model; } 78 \text { had an unfavorable outcome. } \\
\text { †There are } 164 \text { patients }<5 \text { years old included in this model; } 35 \text { had an unfavorable outcome. } \\
\text { †There are } 222 \text { patients } 5-15 \text { years old included in this model; } 43 \text { had an unfavorable outcome. } \\
\text { \$A } 3 \text {-drug regimen includes INH }(\mathrm{H}) \text {, rifampin }(\mathrm{R}) \text {, and pyrazinamide }(\mathrm{Z}) \text {. A 4-drug regimen includes HRZ and ethambutol (E). } \\
\| \text { CD4 count and CD4 percentage are correlated, so to save degrees of freedom only one or the other was used in each regression model. Consistent with their clinical application, } \\
4 \text { percentage was used in the model for those }<5 \text { years old, whereas CD4 count was used for the models including children } 5 \text {-15 years old. }\end{array}$} \\
\hline
\end{tabular}




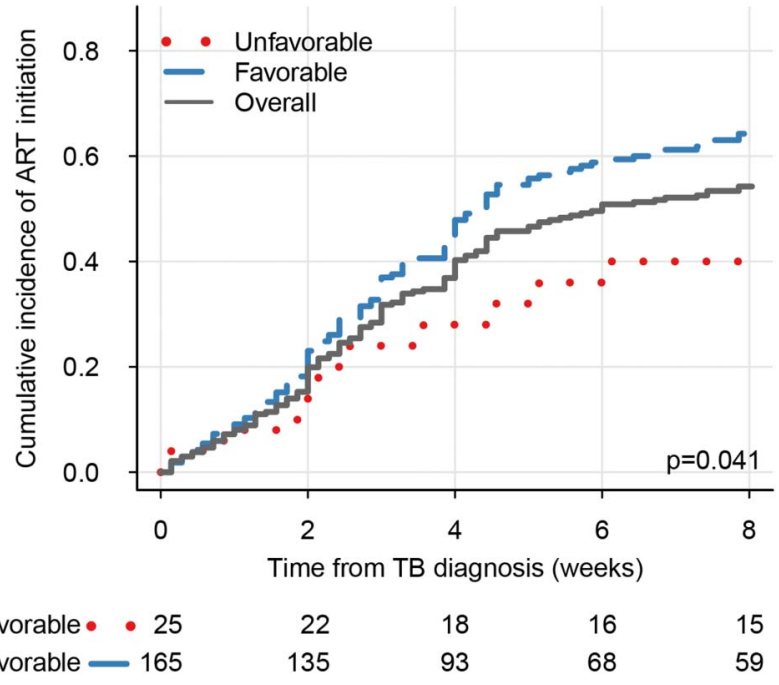

FIGURE 2. Cumulative incidence of ART initiation within 8 weeks after TB diagnosis, among HIV/TB-coinfected children who were previously ART naive and who were alive and retained in care at 8 weeks after TB diagnosis.

WHO makes a "strong recommendation" that a 4-drug IP regimen including ethambutol be used for children from settings where the HIV prevalence is greater than $1 \%$ or there is high prevalence (as defined by national treatment programs) of INH resistance, to minimize the risk of developing or transmitting drug-resistant TB. ${ }^{6,14}$ IP regimen did not have an impact on TB treatment outcomes in this cohort, despite about one-quarter of HIV/TB-coinfected children not receiving ethambutol.

This study adds to the literature that supports early ART as an important part of TB treatment for HIV/TBcoinfected persons, ${ }^{36-39}$ and as such highlights the concerning observation that only half of ART-naive patients in this cohort were started on ART within 8 weeks of TB diagnosis and that $13 \%$ of patients were not started on ART during the study period. Other studies have also reported delays in ART initiation, ${ }^{40}$ and there is some evidence that failing to implement WHO guidelines is associated with substantial mortality. ${ }^{41}$ Further implementation research aimed at improving access to ART for HIV/TB-coinfected children is essential.

Regional differences in outcomes are also important to acknowledge. Patients in the Asia-Pacific and Southern Africa regions were less likely to have unfavorable TB treatment outcomes. These 2 regions also had the highest utilization of sputum induction, gastric aspirates, and NAAT. There were especially high LTFU rates in the Western Africa region, resulting in this region having the highest proportion of unfavorable TB treatment outcomes. It is possible that those who were LTFU may have had different characteristics than those who were classified as having unfavorable outcomes for some other reason. However, due to the relatively small number of unfavorable outcomes, it would be difficult to draw conclusions from sensitivity analyses disaggregating these unfavorable outcomes. Furthermore, other reports have demonstrated significant mortality among those who are LTFU from HIV care and treatment programs. ${ }^{42-44}$ Regardless, improving access to diagnostics and interventions aimed at reducing attrition from care could lead to improved outcomes. In addition, better understanding of the factors that contribute to heterogeneity between sites and regions could result in improved implementation of diagnostic and treatment services.

We were unable to fully assess the appropriateness of ART regimens and modifications to ART regimens in the context of TB treatment, due to the absence of data on weightbased dosing of ART and ATT. The antiretrovirals, nevirapine (an NNRTI) and lopinavir/ritonavir (a boosted PI), significantly interact with the anti-TB medication rifampin and require dose adjustment or changing to an alternative antiretroviral regimen. For those who were treated with nevirapine or lopinavir/ritonavir, it would be important to know whether ART dose adjustments were made. A second limitation was incomplete data for CD4 count/percentage, HIV viral load (75\% missing), WAZ, and ART status. We accounted for incomplete data in our logistic regression models using multiple imputation.

In conclusion, in this large multinational population of HIV/TB-coinfected children, many were not managed as per WHO guidelines. Children with favorable TB outcomes initiated ART sooner than children with unfavorable outcomes. These findings highlight the importance of early ART for children with HIV/TB coinfection, and reinforce the need for implementation research to improve pediatric TB management.

\section{ACKNOWLEDGMENTS}

The authors thank the local and regional IeDEA site staff for their assistance in data collection and quality assurance. They also thank Charlotte Lewden for French translations of case report forms and responses. Sites participating in this project included the following: Sanglah Hospital, Udayana University, Bali, Indonesia; Children's Hospital 1, Ho Chi Minh City, Vietnam; Central University Hospital of Kamenge, Burundi; Kalembelembe Pediatric Hospital, République Démocratique du Congo; Rwanda Military Hospital, Rwanda; Moi University, Moi Teaching and Referral Hospital, Academic Model Providing Access to Healthcare (AMPATH), Eldoret, Kenya; National AIDS Control Programme, Tumbi Regional Hospital, Tanzania; National Institute for Medical Research, Mwanza Research Centre-Kisesa Clinic, Mwanza, Tanzania; Masaka Regional Hospital, Masaka, Uganda; Rahima Moosa Mother and Child Hospital, South Africa; Centre Intégré de Recherches Biocliniques d'Abidjan, Côte d'Ivoire; Centre Hospitalier Universitaire de Cocody, Côte d'Ivoire; and Korle-Bu Teaching Hospital, Accra, Ghana. They also acknowledge the IeDEA TB working group members.

\section{REFERENCES}

1. World Health Organization. Global Tuberculosis Report. 2015. Available at: http://apps.who.int/iris/bitstream/10665/191102/1/9789241565059_eng. pdf?ua $=1$. Accessed June 29, 2016 
2. Corbett EL, Watt CJ, Walker N, et al. The growing burden of tuberculosis: global trends and interactions with the HIV epidemic. Arch Intern Med. 2003;163:1009-1021.

3. Fenner L, Brinkhof MW, Keiser O, et al. Early mortality and loss to follow-up in HIV-infected children starting antiretroviral therapy in Southern Africa. J Acquir Immune Defic Syndr. 2010;54:524-532.

4. World Health Organization. Definitions and Reporting Framework for Tuberculosis-2013 Revision. Available at: http://apps.who.int/iris/ bitstream/10665/79199/1/9789241505345_eng.pdf. Accessed July 6, 2016.

5. Turkova A, Chappell E, Chalermpantmetagul S, et al. Tuberculosis in HIV-infected children in Europe, Thailand and Brazil: paediatric TB: HIV EuroCoord study. Poster presented at: 20th International Workshop on HIV and Hepatitis Observational Databases; April, 2016; Budapest, Hungary.

6. World Health Organization. Guidance for National Tuberculosis Programmes on the Management of Tuberculosis in Children-2nd Edition. 2014. Available at: http://apps.who.int/medicinedocs/documents/ s21535en/s21535en.pdf. Accessed June 29, 2016.

7. Donald PR, Maher D, Qazi S. A research agenda to promote the management of childhood tuberculosis within national tuberculosis programmes. Int J Tuberc Lung Dis. 2007;11:370-380

8. Geoghagen M, Farr JA, Hambleton I, et al. Tuberculosis and HIV co-infections in Jamaican children. West Indian Med J. 2004;53: 339-345.

9. Graham SM, Gie RP, Schaaf HS, et al. Childhood tuberculosis: clinical research needs. Int J Tuberc Lung Dis. 2004;8:648-657.

10. Hicks RM, Padayatchi N, Shah NS, et al. Malnutrition associated with unfavorable outcome and death among South African MDR-TB and HIV co-infected children. Int J Tuberc Lung Dis. 2014;18:1074-1083.

11. Jeena PM, Pillay P, Pillay T, et al. Impact of HIV-1 co-infection on presentation and hospital-related mortality in children with culture proven pulmonary tuberculosis in Durban, South Africa. Int $J$ Tuberc Lung Dis. 2002;6:672-678.

12. Detjen AK, DiNardo AR, Leyden J, et al. Xpert MTB/RIF assay for the diagnosis of pulmonary tuberculosis in children: a systematic review and meta-analysis. Lancet Respir Med. 2015;3:451-461.

13. Thee S, Detjen A, Quarcoo D, et al. Ethambutol in paediatric tuberculosis: aspects of ethambutol serum concentration, efficacy and toxicity in children. Int $J$ Tuberc Lung Dis. 2007;11:965-971.

14. World Health Organization. Ethambutol Efficacy and Toxicity: Literature Review and Recommendations for Daily and Intermittent Dosage in Children. 2006. Available at: http://apps.who.int/iris/bitstream/10665/ 69366/1/WHO_HTM_TB_2006.365_eng.pdf. Accessed July 13, 2016.

15. Cotton MF, Rabie H, van Zyl GU. Antiretroviral therapy in children with tuberculosis: progress toward defining the issues. J Infect Dis. 2010;201: $1113-1114$.

16. Marais BJ, Gie RP, Hesseling AC, et al. A refined symptom-based approach to diagnose pulmonary tuberculosis in children. Pediatrics. 2006;118:e1350-e1359.

17. Marais BJ, Graham SM, Cotton MF, et al. Diagnostic and management challenges for childhood tuberculosis in the era of HIV. $J$ infect dis. 2007;196(suppl 1):S76-S85.

18. Newton SM, Brent AJ, Anderson S, et al. Paediatric tuberculosis. Lancet Infect Dis. 2008;8:498-510.

19. Ballif M, Renner L, Claude Dusingize J, et al. Tuberculosis in pediatric antiretroviral therapy programs in low- and middle-income countries: diagnosis and screening practices. J Pediatr Infect Dis Soc. 2015;4:30-38.

20. Link-Gelles R, Moultrie H, Sawry S, et al. Tuberculosis immune reconstitution inflammatory syndrome in children initiating antiretroviral therapy for HIV infection: a systematic literature review. Pediatr Infect Dis J. 2014;33:499-503

21. Van Rie A, Sawry S, Link-Gelles R, et al. Paradoxical tuberculosisassociated immune reconstitution inflammatory syndrome in children. Pediatr Pulmonol. 2016;51:157-164.

22. Mori AT, Owenya J. Stock-outs of antiretroviral drugs and coping strategies used to prevent changes in treatment regimens in Kinondon District, Tanzania: a cross-sectional study. J Pharm Policy Pract. 2014;7:3.

23. Pasquet A, Messou E, Gabillard D, et al. Impact of drug stock-outs on death and retention to care among HIV-infected patients on combination antiretroviral therapy in Abidjan, Cote d'Ivoire. PloS One. 2010;5:e13414.
24. Schouten EJ, Jahn A, Ben-Smith A, et al. Antiretroviral drug supply challenges in the era of scaling up ART in Malawi. J Int AIDS Soc. 2011; 14(suppl 1):S4

25. Bouazza N, Foissac F, Fauchet F, et al. Lopinavir/ritonavir plus lamivudine and abacavir or zidovudine dose ratios for paediatric fixeddose combinations. Antivir Ther. 2015;20:225-233.

26. Howard LM, Tique JA, Gaveta S, et al. Health literacy predicts pediatric dosing accuracy for liquid zidovudine. AIDS. 2014;28:1041-1048.

27. Taneja R, Garcia-Prats AJ, Furin J, et al. Paediatric formulations of second-line anti-tuberculosis medications: challenges and considerations. Int J Tuberc Lung Dis. 2015;19(suppl 1):61-68.

28. Vitoria M, Ford N, Doherty M, et al. Simplification of antiretroviral therapy: a necessary step in the public health response to HIV/AIDS in resource-limited settings. Antivir Ther. 2014;19(suppl 3):31-37.

29. Egger M, Ekouevi DK, Williams C, et al. Cohort profile: the international epidemiological databases to evaluate AIDS (IeDEA) in sub-Saharan Africa. Int J Epidemiol. 2012;41:1256-1264.

30. Harris PA, Taylor R, Thielke R, et al. Research electronic data capture (REDCap)-a metadata-driven methodology and workflow process for providing translational research informatics support. $J$ Biomed Inform. 2009:42:377-381

31. World Health Organization. Consolidated Guidelines on the Use of Antiretroviral Drugs for Treating and Preventing HIV Infection: Recommendations for a Public Health Approach—2nd Edition. 2016. Available at: http://www.who.int/entity/hiv/pub/arv/arv-2016/en/index. html. Accessed July 6, 2016.

32. Flegal KM, Cole TJ. Construction of LMS parameters for the Centers for Disease Control and Prevention 2000 growth charts. Natl Health Stat Report. 2013;63:1-3.

33. Mishra AK, Driessen NN, Appelmelk BJ, et al. Lipoarabinomannan and related glycoconjugates: structure, biogenesis and role in Mycobacterium tuberculosis physiology and host-pathogen interaction. FEMS Microbiol Rev. 2011;35:1126-1157.

34. Pai M, Schito M. Tuberculosis diagnostics in 2015: landscape, priorities, needs, and prospects. $J$ Infect Dis. 2015;211(suppl 2):S21-S28.

35. World Health Organization. The Use of Lateral Flow Urine Lipoarabinomannan Assay (LF-lam) for the Diagnosis and Screening of Active Tuberculosis in People Living with HIV. 2015. Available at: http://www.who.int/tb/publications/use-of-lf-lam-tb-hiv/en/. Accessed July 1,2016

36. Abay SM, Deribe K, Reda AA, et al. The effect of early initiation of antiretroviral therapy in TB/HIV-coinfected patients: a systematic review and meta-analysis. J Int Assoc Provid AIDS Care. 2015;14:560-570.

37. Manosuthi W, Wiboonchutikul S, Sungkanuparph S. Integrated therapy for HIV and tuberculosis. AIDS Res Ther. 2016;13:22

38. Yan $\mathrm{S}, \mathrm{Chen} \mathrm{L}, \mathrm{Wu} \mathrm{W}$, et al. Early versus delayed antiretroviral therapy for HIV and tuberculosis co-infected patients: a systematic review and meta-analysis of randomized controlled trials. PloS One. 2015:10 e0127645.

39. Yotebieng M, Van Rie A, Moultrie H, et al. Effect on mortality and virological response of delaying antiretroviral therapy initiation in children receiving tuberculosis treatment. AIDS. 2010;24:1341-1349.

40. Patel MR, Nana M, Yotebieng M, et al. Delayed antiretroviral therapy despite integrated treatment for tuberculosis and HIV infection. Int $J$ Tuberc Lung Dis. 2014;18:694-699.

41. Patel MR, Westreich D, Yotebieng M, et al. The impact of implementation fidelity on mortality under a CD4-stratified timing strategy for antiretroviral therapy in patients with tuberculosis. Am $J$ Epidemiol. 2015;181:714-722.

42. Cornell M, Lessells R, Fox MP, et al. Mortality among adults transferred and lost to follow-up from antiretroviral therapy programmes in South Africa: a multicenter cohort study. J Acquir Immune Defic Syndr. 2014; 67:e67-e75.

43. Geng EH, Odeny TA, Lyamuya RE, et al. Estimation of mortality among HIV-infected people on antiretroviral treatment in East Africa: a sampling based approach in an observational, multisite, cohort study. Lancet HIV. 2015;2:e107-e116.

44. Schoni-Affolter F, Keiser O, Mwango A, et al. Estimating loss to followup in HIV-infected patients on antiretroviral therapy: the effect of the competing risk of death in Zambia and Switzerland. PloS One. 2011;6: e27919. 